\title{
MACROINVERTEBRADOS BENTÓNICOS Y CALIDAD DEL AGUA EN UN TRAMO DEL RÍO BOGOTÁ. CAJICÁ-COLOMBIA
}

\section{BENTHIC MACROINVERTEBRATES AND WATER QUALITY IN A STRETCH OF THE RIVER BOGOTA. CAJICA-COLOMBIA}

\author{
Diego Fernando Ramírez ${ }^{1}$, Grace Margarita Talero², Raúl Hernando López ${ }^{3}$ \\ 1,2 Biólogos. Asistentes de Investigación. ${ }^{1}$ dframireza@gmail.com; ${ }^{2}$ margarita.talmar@gmail.com; ${ }^{3}$ Biólogo Marino, Dr. rer. \\ nat. Profesor Asociado. Universidad Militar Nueva Granada. Campus Nueva Granada Km 2 vía Cajicá-Zipaquirá. Cundinamarca, \\ Colombia.raul.lopez@unimilitar.edu.co
}

Rev. U.D.C.A Act. \& Div. Cient. 16(1): 205 - 214, 2013

\section{RESUMEN}

Entre febrero y septiembre de 2011, se tomaron muestras de sustrato blando en aguas someras, en tres sectores de un tramo rural del río Bogotá, ubicado en el área de Cajicá (Cundinamarca). Dada la importancia del río para la Sabana de Bogotá, el objetivo fue evaluar la calidad del agua, con base en algunas variables abióticas, los macroinvertebrados bentónicos y los índices BMWP/Col. y ASPT. No se observaron diferencias importantes entre los tres sectores de muestreo, pero sí variaciones cronológicas, asociadas a la influencia de las lluvias, intensificadas por un episodio moderado de La Niña. De este modo, las precipitaciones y el perfil limnológico, se relacionaron con la abundancia y la composición taxonómica de los invertebrados. Un sustrato arenoso-limoso favoreció la abundancia de Tubificidae $(72,12 \%)$ y Chironomidae $(23,43 \%)$, por su adaptabilidad a cargas orgánicas y de detritus altas y concentraciones bajas de $\mathrm{O}_{2}$. Estos taxa, junto con Physidae, Glossiphoniidae y Tipulidae, principalmente, indicaron contaminación moderada en los tres sectores (BMWP/Col. $\mathrm{A}=48, \mathrm{~B}=48$, $C=43$ ) y eutrofización, como consecuencia del impacto humano (aguas residuales y actividades agropecuarias), que afectan las aguas del río y su biota.

Palabras clave: Río Bogotá, BMWP/Col., contaminación, sedimentos, bentos.

\section{SUMMARY}

From February to September 2011, in shallow waters in three sections of a rural stretch of the Bogota River, located at Cajica (Cundinamarca), some soft substrate samples were taken. Given the importance of the river to the savannah of Bogota, the objective was to evaluate the water quality, based on some environmental variables, the benthic macroinvertebrates and the BMWP/col. and ASPT indexes. No significant differences were observed among the three sampling sections, but chronological variations related to the influence of rainfall, enhanced by a moderate La Niña episode were detected. Thus, precipitations and the limnological profile were associated with the abundance, biomass and taxonomic composition of the invertebrates. A sandy-loam substrate favored the density and biomass of Tubificiade $\left(72.12 \%, 0.15 \mathrm{~g} / \mathrm{m}^{2}\right)$ and Chironomidae $(23.43 \%$, $0.23 \mathrm{~g} / \mathrm{m}^{2}$ ), due to their adaptability to high concentration of organic matter and detritus and low $\mathrm{O}_{2}$ values. These taxa and mainly Physidae, Glossiphoniidae, and Tipulidae indicated moderate pollution in the three sections (BMWP/ Col. $A=48, B=48, C=43$ ) and eutrophication, as a result of human impact (wastewater and domestic discharges, and farming activities), affecting the river and its biota.

Key words: Bogotá River, BMWP/Col., pollution, sediments, benthos.

\section{INTRODUCCIÓN}

Los sistemas lóticos, fluviales o de aguas corrientes (arroyos, quebradas y ríos), se diferencian de otros ecosistemas acuáticos por el continuo y rápido caudal de sus aguas, que afectan los flujos de energía y de materia orgánica, la generación de biodiversidad, las sucesiones ecológicas y el mantenimiento de los equilibrios ecológicos (Wetzel, 2001; Roldán \& Ramírez, 2008). 
Los macroinvertebrados $(\geq 500 \mu \mathrm{m})$ acuáticos son principalmente bentónicos, i.e., que habitan en o sobre el fondo, donde participan en la recirculación de nutrientes y en la degradación de materia orgánica (Riss et al. 2002; Roldán, 2003). En los sistemas lóticos, su distribución longitudinal es muy importante (Jones, 2011), pues se han observado cambios, incluso altitudinales de biodiversidad asociados al clima y a la topografía de las cuencas (Jacobsen, 2003; Goitia \& Bustamante, 2009).

El río Bogotá recoge las aguas de una cuenca de $6000 \mathrm{~km}^{2}$ y es uno de los sistemas lóticos más relevantes para el desarrollo de la Sabana de Bogotá, aunque debido a las actividades agrícolas, mineras, industriales y ganaderas, su calidad se ha deteriorado severamente (CAR-Cundinamarca, 2006). Muy pocos trabajos de la calidad del agua se han efectuado en el área de estudio (e.g., Sierra et al. 2002; Gaviria et al. 2004), a diferencia de otras regiones de Colombia, donde se han empleado macroinvertebrados acuáticos (e.g., Lozano, 2005; García et al. 2007; Liévano \& Ospina, 2007; Bustamante et al. 2008; Walteros \& Paiba, 2010; Marín et al. 2011; Montoya et al. 2011).

Uno de los índices empleados para el diagnóstico de los cuerpos de agua es el BMWP (Biological Monitoring Working Party), adaptado para Colombia (BMWP/Col.), que se basa en algunas familias de macroinvertebrados acuáticos. El índice se da por la suma total de la puntuación de cada familia (1-10) y define clases, calidad, puntaje y categorías, así: I= buena, >150/101-130), muy limpia; $\mathrm{II}=$ II aceptable, 61-100, ligeramente contaminada, III= dudosa, 36-60, moderadamente contaminada; IV= crítica, 16-35, muy contaminada; $V=$ muy crítica, $<15$, fuertemente contaminada (Roldán, 1996; 2003).

El índice ASPT (Average Score Per Taxon= Puntaje promedio por Taxon $=1-10)$, se obtiene al dividir el BMWP, por el número de taxa incluidos en el cálculo (Armitage et al. 1983; Walley \& Hawkes, 1997).

El propósito de este trabajo fue valorar la calidad del agua aplicando estos dos índices, con base en algunas variables físico-químicas y su influencia sobre la comunidad de macroinvertebrados bentónicos, en un tramo de la cuenca alta del río Bogotá.

\section{MATERIALES Y MÉTODOS}

Se estudió el tramo del río Bogotá limítrofe con el Campus de la Universidad Militar Nueva Granda (Campus-UMNG), ubicado a 2558 msnm en la salida rural norte del municipio de Cajicá (Cundinamarca), con clima frío (promedio $13^{\circ} \mathrm{C}$ ), semiseco, característico de la cuenca alta del río Bogotá, afectada por descargas de residuos de pesticidas, agroquímicos y aguas negras. Las precipitaciones $(<1000 \mathrm{~mm})$ tienen un régimen bimodal (máx. abril-mayo y septiembre-noviembre; mín. diciembre-marzo y julio-agosto), influenciado por los eventos El Niño y La Niña (CAR-Cundinamarca, 2006).

Los sectores de muestreo, se ubicaron en un meandro del río influenciado por cultivos de flores y un campo de golf $y$ se designaron como: A: 456’33.78" N-7400'27.10”O, B: 456’25.02" N-7400'17.50”O y C: $4^{\circ} 57^{\prime} 33.78$ "N-7400'28.10”O (Figura1).

Abarcando periodos de diferente intensidad pluviométrica, en cada sector se hicieron muestreos de sustrato blando, original y tres réplicas, en aguas someras $(10-20 \mathrm{~cm})$ cerca de la orilla, con una red Surber de $25 \times 25 \mathrm{~cm}$, con malla de $250 \mu \mathrm{m}$, la cual, se ubicó contracorriente. En todos los casos, se removieron los primeros $5-10 \mathrm{~cm}$ de sustrato, mediante una pala graduada $(\mathrm{cm})$, se depositaron en bolsas de polietileno y se fijaron con alcohol al 70\% (Oyague, 2009). La continuidad de los muestreos no fue posible, pues no siempre se pudo acceder a los lugares evaluados, debido a la influencia de un evento La Niña, con precipitaciones fuertes e inundaciones más intensas en la cordillera occidental y la Sabana de Bogotá (120\% por encima del promedio) (IDEAM, 2011). Entonces, solamente se consideraron las fechas: 08 de febrero, 29 de marzo, 11 de julio, 01 y 29 de agosto, y 26 de septiembre de 2011.

En cada sector, se emplearon sondas Schott ${ }^{\circledR}$, para las mediciones en la superficie de temperatura $\left({ }^{\circ} \mathrm{C}\right), \mathrm{pH}$ (unidades de $\mathrm{pH})$, conductividad $(\mu \mathrm{S} / \mathrm{cm})$ y $\mathrm{O}_{2}$ disuelto (mg/L); además, un fotómetro Visocolor Macherey-Nagel $®$, para estimar las concentraciones de $\mathrm{NO}_{2}{ }^{-}, \mathrm{NO}_{3}{ }^{-}, \mathrm{NH}_{4}{ }^{+}, \mathrm{PO}_{4}{ }^{3-}$ y $\mathrm{Fe}^{2+}$ (mg/L). La dureza total $(\mathrm{mg} / \mathrm{L})$, se valoró con un juego de campo Visocolor ${ }^{\circledR}$ y la transparencia $(\mathrm{cm})$ con un disco Secchi.

Las muestras de sustrato se secaron hasta peso constante, en un horno a $105^{\circ} \mathrm{C}$; luego, se pasaron por tamices de 250 , 53 y $45 \mu \mathrm{m}$, para tipificarlos, según la escala de Wentworth (Wetzel, 2001). El mismo procedimiento de secado, se aplicó a las muestras de $50 \mathrm{~mL}$ de agua, tomadas en cada fecha y sector, para estimar los sólidos disueltos, suspendidos y totales (mg/L), filtrando el agua con papel de fibra de vidrio (1,5 $\mu \mathrm{m})$ (Roldán \& Ramírez, 2008). En todos los casos, se empleó una balanza analítica OHAUS-Explorer®.

Los macroinvertebrados, se separaron del sedimento con un tamiz de $60 \mu \mathrm{m}$, para su cuantificación e identificación (Merrit \& Cummins, 1984; Roldán, 1996, 2003; Liévano \& Ospina, 2007; Mugnai et al. 2010), bajo un estereoscopio y un microscopio Carl Zeiss Stemi DV4 y Axiostar Plus, respectivamente.

En la evaluación del cuadro limnológico general, en relación 

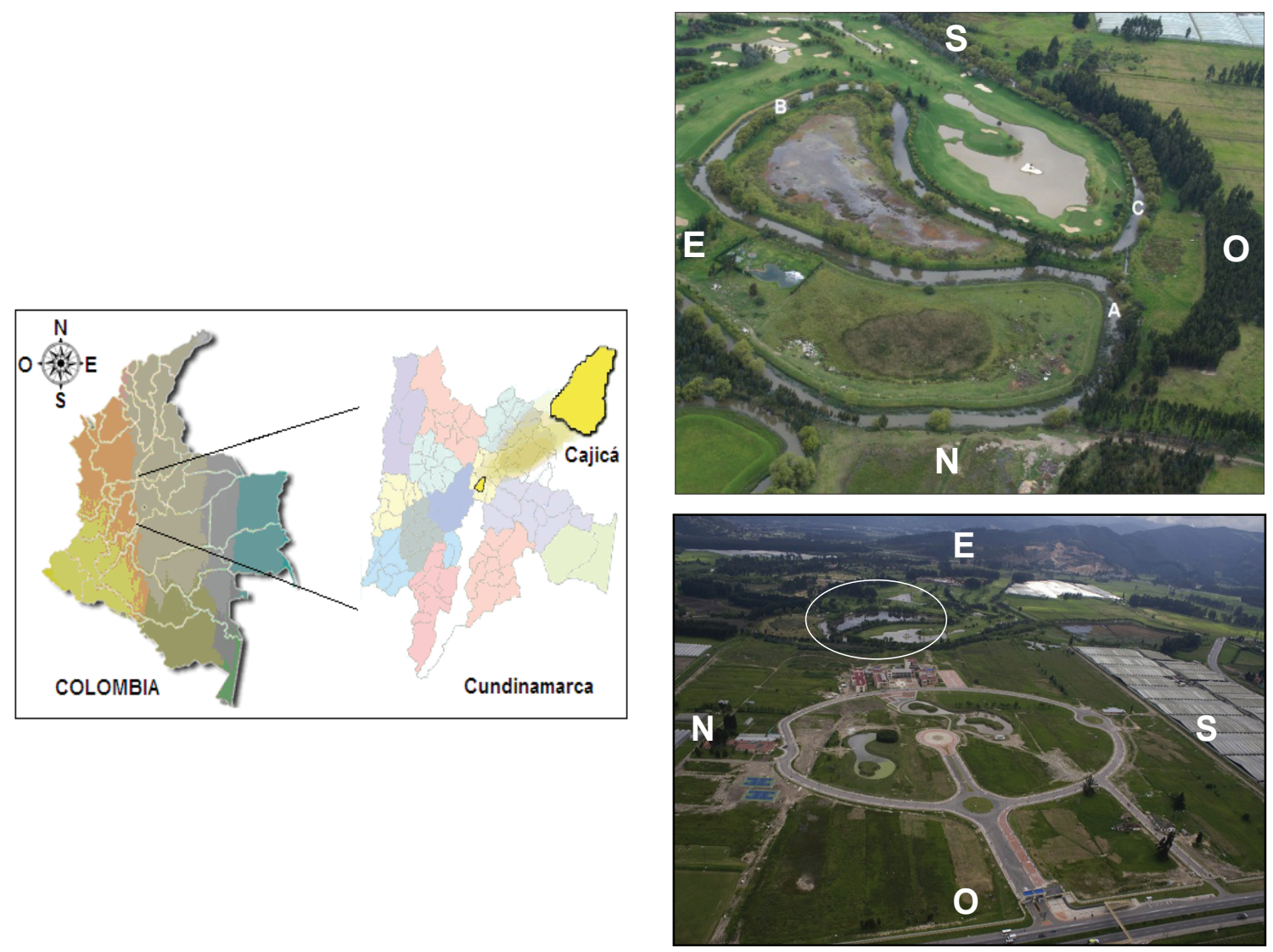

Figura 1. Localización del área (modificado de www.cajica-cundinamarca.gov.co) y los sectores de estudio (A, B, C), en el Campus-UMNG y el río Bogotá (Cajicá, Cundinamarca).

con los invertebrados bentónicos, se utilizaron los índices BMWP-Col. (Datos de un solo individuo no se incluyeron en el cálculo) (Roldán, 1996, 2003) y ASPT (Armitage et al. 1983; Walley \& Hawkes, 1997) y un Análisis de Correspondencia Canónica (ACC). Los valores de precipitación fueron suministrados por la estación meteorológica del CampusUMNG y la estación hidrometeorológica ALCO de la Corporación Autónoma Regional (CAR)-Cundinamarca (sin datos para enero-febrero de 2011). El Coeficiente de Variación (CV) sirvió para verificar la dispersión de los datos con respecto al valor medio y comprobar el grado de homogeneidad.

Ya que a la fecha no se encuentran definidos los criterios de calidad para el uso de las aguas en Colombia (Decreto 3930 de 2010, Capítulo V, Artículo 20), cuando fue el caso se tuvieron en cuenta los límites establecidos en el Decreto 1594 de 1984 (MINAMBIENTE, 2011).

\section{RESULTADOS Y DISCUSIÓN}

Comunidad de macroinvertebrados acuáticos-Índices BMWPCol. y ASPT: Se capturaron 9559 individuos distribuidos en tres phyla, seis clases, 10 órdenes y 17 familias; la mayoría perteneció a las familias Tubificidae (Annelida) $(72,12 \%)$ y Chironomidae (Diptera) (23,43\%). En los tres sectores, los índices BMWP/Col. $(A=48, B=48, C=43$ ) y ASPT (4 en los tres) (Tabla 1) revelaron una contaminación moderada del agua (dudosa=Clase III) (Roldán, 2003), durante el periodo evaluado. En este diagnóstico, posiblemente, influyeron los aportes de aguas residuales aguas arriba y la concentración de sedimentos, productos químicos y materia orgánica, dados por las actividades agropecuarias e industriales en cercanías al río (Gaviria et al. 2004; CAR-Cundinamarca, 2006).

Tubificidae y Chironomidae, así como Physidae, Glossiphoniidae y Tipulidae, también halladas en este estudio, son indicadoras de aguas mesotróficas a eutróficas, contaminadas a muy contaminadas por alto impacto antrópico, por su mayor abundancia y porque su presencia está favorecida por su tolerancia a poco $\mathrm{O}_{2}$ y disponibilidad alta de detritus orgánico, que limitan la cantidad de familias en los cuerpos de agua (Roldán, 1996, 2003; Liévano \& Ospina, 2007). Esto complementa la calificación de aguas moderadamente contaminadas e, incluso, eutrofizadas del tramo del río Bogotá. 
Tabla 1. Ubicación taxonómica y abundancia de macroinvertebrados, BMWP/Col. (Roldán, 2003) y ASPT (Walley \& Hawkes, 1997) en el tramo del río Bogotá evaluado. *No considerada. **Sin valor de indicación (Roldán, 2003). ***Ausente.

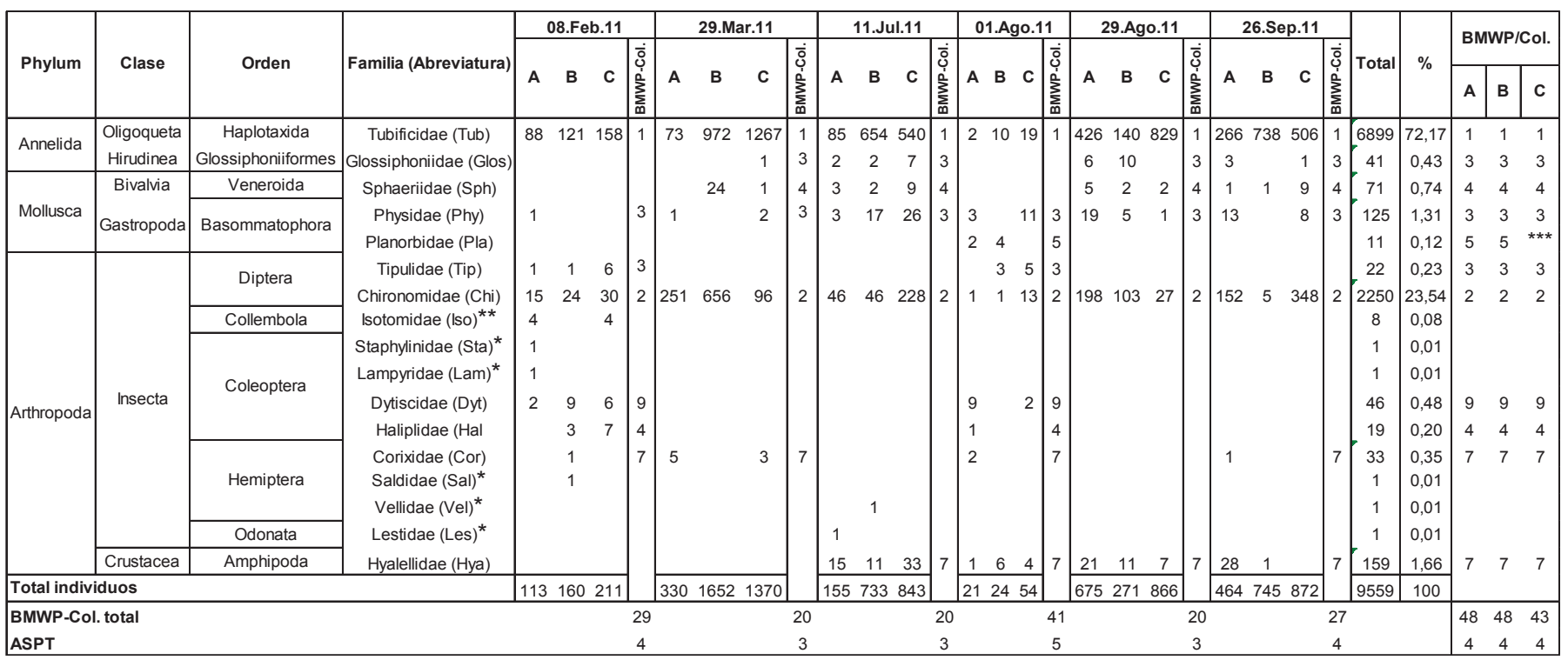

Se puede mencionar la semejanza taxonómica y en número de familias (11-19) con varias quebradas y ríos de otras regiones de Colombia (e.g., Caicedo \& Palacio, 1998; Lozano, 2005; García et al. 2007; Arango et al. 2008; Walteros \& Paiba,
2010) y, en otros casos, mayor riqueza de familias (23-72) (e.g., Sánchez, 2005; Liévano \& Ospina, 2007; Arango et al. 2008; Bustamante et al. 2008; Castellanos \& Serrato, 2008; Montoya, 2008; Walteros \& Paiba, 2010; Marín et al. 2011;

Tabla 2. Algunos sistemas lóticos colombianos en que se han efectuado investigaciones sobre macroinvertebrados bentónicos.

\begin{tabular}{|c|c|c|c|c|c|c|c|}
\hline Departamento & Nombre & Tipo & Sustrato & Red/otros & Familias & BMWP/Col. & Autores \\
\hline \multirow{7}{*}{ Antioquia } & La Mosca & \multirow{5}{*}{ quebrada } & arenoso, pedregoso, fango & Surber & 16 & no & Caicedo \& Palacio (1998) \\
\hline & La Cristalina & & \multirow{2}{*}{ rocoso } & \multirow{2}{*}{ D-net } & 27 & \multirow{2}{*}{ si } & \multirow{2}{*}{ Arango et al. (2008) } \\
\hline & La Risaralda & & & & 19 & & \\
\hline & Los Andes & & sin especificación & $\begin{array}{c}\text { Pantalla, triangular, } \\
\text { deriva, manual }\end{array}$ & 52 & si & Montoya (2008) \\
\hline & La Popala & & grava, arena, hojarasca & Surber & 23 & BMWP & Milán et al. (2011) \\
\hline & Negro & río & \begin{tabular}{|c|} 
piedras, gravas, cantos \\
rodados, macrófitas, perifiton \\
piedras, fragmentos de \\
concreto, fango \\
\end{tabular} & $\begin{array}{l}\text { D-net, pantalla, } \\
\text { surber, manual, } \\
\text { draga Ekman }\end{array}$ & 31 & \multirow{6}{*}{ si } & Montoya et al. (2011) \\
\hline & Las Cimarronas & quebrada & sin especificación & $\begin{array}{c}\text { pantalla, triangular, } \\
\text { manual }\end{array}$ & 62 & & \\
\hline Bogotá & Juan Amarillo & río & sin especificación & Surber & 20 & & Lozano (2005) \\
\hline \multirow[t]{3}{*}{ Caldas } & La Mula & \multirow[t]{2}{*}{ quebrada } & \multirow{3}{*}{$\begin{array}{c}\text { sustratos rocosos, orillas con } \\
\text { vegetación, } \\
\text { sedimento fino, macrófitos, } \\
\text { detritus }\end{array}$} & \multirow[t]{3}{*}{ D-net, Surber } & 18 & & \multirow[t]{3}{*}{ Walteros \& Paiba (2010) } \\
\hline & El Diamante & & & & 24 & & \\
\hline & Chinchiná & \multirow{5}{*}{ río } & & & 24 & & \\
\hline Cundinamarca & Bahamón & & sin especificación & No especifica & 30 & BMWP & Liévano \& Ospina (2007) \\
\hline \multirow[b]{2}{*}{ Norte de Santander } & El Mortiño & & sin especificación & Surber & 30 & no & Castellanos \& Serrato (2008) \\
\hline & Pamplonita & & $\begin{array}{c}\text { pedregoso, arenoso, } \\
\text { vegetación circundante }\end{array}$ & Pantalla, D-net & 72 & BMWP & Sánchez (2005) \\
\hline \multirow{4}{*}{ Quindío } & Quindío & & $\begin{array}{l}\text { rocas, arena, vegetación } \\
\text { ribereña, troncos, hojarasca }\end{array}$ & $\begin{array}{l}\text { D-net, pinzas } \\
\text { entomológicas }\end{array}$ & 38 & si & Bustamante et al. (2008) \\
\hline & Villa Paola & \multirow{3}{*}{ quebrada } & sinespecificación & D-net manual & 11 & \multirow{3}{*}{ no } & \multirow{2}{*}{ García et al. (2007) } \\
\hline & Doña Juana & & & D-hes, manual & 13 & & \\
\hline & La Florida & & rocoso, vegetal & manual & 26 & & Marín et al. (2011) \\
\hline
\end{tabular}


Milán et al. 2011; Montoya et al. 2011; García et al. 2012) (Tabla 2).

También existen grandes diferencias entre esas investigaciones y los resultados de este estudio, los cuales, indudablemente se relacionan con las diferentes metodologías, duración y frecuencia de los muestreos, número de sectores considerados, hábitats, tipo de sustrato y las características de cada sistema fluvial.

Aspectos físicos y químicos (Tabla 3). Las diferencias en los promedios de la mayoría de variables abióticas fueron mínimas, dada la cercanía entre los puntos, como lo soportan los CV. Las lluvias abundaron en abril-mayo de 2011, por encima de los promedios anuales de los últimos veinte años, debido al episodio moderado de La Niña 2011 (IDEAM, 2011); fueron mínimas entre junio y octubre, para aumentar los últimos meses, conforme al régimen bimodal en el área de estudio (CAR-Cundinamarca, 2006). Estas variaciones cronológicas debieron incidir en las variables consideradas, pues las lluvias acrecientan las escorrentías, el caudal de los cuerpos lóticos y alteran la composición físicoquímica del agua (Wetzel, 2001); cuando son más fuertes (periodos de altas) aumentan el arrastre de sedimentos (Gaviria et al. 2004; CAR-Cundinamarca, 2006) y la deriva de macroinvertebrados (Rodríguez et al. 2007), que se restringe en periodos de bajas con la disminución de sólidos disueltos, conductividad y turbidez (García et al. 2007). De hecho, en el presente estudio las abundancias se incrementaron con la pluviosidad.

La transparencia $(\mathrm{cm})$ varió entre 13 (septiembre) y 44 (abril), en lo que intervienen el detritus orgánico y los sólidos de procesos erosivos (APHA et al. 2005). Los promedios de

Tabla 3. Datos abióticos en los sectores de estudio del río Bogotá. Pre = precipitaciones, Tra $=$ transparencia, $\mathrm{SS}=$ sólidos suspendidos, $\mathrm{SD}=$ sólidos disueltos, $\mathrm{ST}=$ sólidos totales, $\mathrm{Con}=$ conductividad, $\mathrm{Tem}=$ temperatura, $\mathrm{DT}=$ dureza total.

\begin{tabular}{|c|c|c|c|c|c|c|c|c|c|c|c|c|c|c|c|}
\hline \multicolumn{16}{|c|}{ Sector A } \\
\hline Fecha & Pre. & Tra. & & SD & ST & Con & Tem. & $\mathrm{pH}$ & $\mathrm{O}_{2}$ & $\mathrm{NH}_{4}{ }^{+}$ & $\mathrm{NO}_{2}^{-}$ & $\mathrm{NO}_{3}^{-}$ & $\mathrm{PO}_{4}{ }^{3-}$ & $\mathrm{Fe}^{2+}$ & DT \\
\hline & $\mathrm{mm}$ & $\mathrm{cm}$ & $\mathrm{mg} / \mathrm{L}$ & $\mathrm{mg} / \mathrm{L}$ & $\mathrm{mg} / \mathrm{L}$ & $\mu \mathrm{Scm}^{-1}$ & ${ }^{\circ} \mathrm{C}$ & & $\mathrm{mg} / \mathrm{L}$ & $\mathrm{mg} / \mathrm{L}$ & $\mathrm{mg} / \mathrm{L}$ & $\mathrm{mg} / \mathrm{L}$ & $\mathrm{mg} / \mathrm{L}$ & $\mathrm{mg} / \mathrm{L}$ & $\mathrm{mg} / \mathrm{L}$ \\
\hline 08.Feb.11 & 0,0 & 23,9 & 0,0 & 0,0 & 0,0 & 291,0 & 17,1 & 6,5 & 1,0 & 0,5 & 0,1 & 4,0 & 0,4 & 0,0 & 1,3 \\
\hline 29.Marz.11 & 46,3 & 16,8 & 580,0 & 347,5 & 927,5 & 695,0 & 17,8 & 6,6 & 1,5 & 1,8 & 0,1 & 4,0 & 3,4 & 0,4 & 1,3 \\
\hline 11.Jul.11 & 11,9 & 44,0 & 560,0 & 109,0 & 669,0 & 218,0 & 16,0 & 6,4 & 6,8 & 1,4 & 0,0 & 4,0 & 0,6 & 0,5 & 2,2 \\
\hline 01.Ago.11 & 20,0 & 13,0 & 80,0 & 97,0 & 177,0 & 194,0 & 15,9 & 6,4 & 7,8 & 1,4 & 0,1 & 0,4 & 0,6 & 0,9 & 2,0 \\
\hline 29.Ago.11 & 0,0 & 26,0 & 60,0 & 126,5 & 186,5 & 253,0 & 17,0 & 6,1 & 5,7 & 0,5 & 0,3 & 5,0 & 0,6 & 0,1 & 2,2 \\
\hline 26.Sep.11 & 1,4 & 34,0 & 100,0 & 236,0 & 336,0 & 472,0 & 17,6 & 5,7 & 5,3 & 0,4 & 0,0 & 1,0 & 0,3 & 0,4 & 2,4 \\
\hline Promedio & 13,3 & 26,3 & 230,0 & 152,7 & 382,7 & 353,8 & 16,9 & 6,3 & 4,7 & 1,0 & 0,1 & 3,1 & 1,0 & 0,4 & 1,9 \\
\hline $\mathrm{CV}$ & 1,4 & 0,4 & 1,2 & 0,8 & 0,9 & 0,5 & 0,0 & 0,1 & 0,6 & 0,6 & 0,9 & 0,6 & 1,2 & 0,9 & 0,3 \\
\hline \multicolumn{16}{|c|}{ Sector B } \\
\hline 08.Feb.11 & 0,0 & 34,3 & 0,0 & 0,0 & 0,0 & 360,0 & 17,7 & 6,8 & 0,5 & 1,0 & 0,1 & 4,0 & 1,1 & 0,0 & 2,2 \\
\hline 29.Marz.11 & 46,3 & 21,7 & 140,0 & 340,0 & 480,0 & 680,0 & 17,9 & 6,6 & 1,8 & 3,1 & 0,1 & 4,0 & 4,4 & 0,4 & 3,0 \\
\hline 11.Jul.11 & 11.9 & 35,3 & 480,0 & 109,5 & 589,5 & 219,0 & 16,9 & 6,6 & 7,1 & 0,5 & 0,1 & 4,0 & 0,7 & 0,4 & 1,4 \\
\hline 01.Ago.11 & 20,0 & 13,5 & 40,0 & 97,0 & 137,0 & 194,0 & 16,0 & 6,2 & 7,8 & 1,4 & 0,1 & 0,4 & 0,7 & 0,5 & 1,8 \\
\hline 29.Ago.11 & 0,0 & 30,0 & 100,0 & 109,0 & 209,0 & 218,0 & 16,8 & 5,2 & 5,6 & 0,7 & 0,2 & 4,0 & 0,7 & 0,4 & 2,2 \\
\hline 26.Sep.11 & 1,4 & 28,0 & 140,0 & 174,5 & 314,5 & 349,0 & 18,1 & 6,8 & 7,4 & 0,4 & 0,0 & 1,0 & 0,4 & 0,3 & 2,8 \\
\hline Promedio & 13,5 & 27,1 & 150,0 & 138,3 & 288,3 & 336,7 & 17,2 & 6,4 & 5,0 & 1,2 & 0,1 & 2,9 & 1,3 & 0,3 & 2,2 \\
\hline CV & 1,4 & 0,3 & 1,1 & 0,8 & 0,8 & 0,5 & 0,0 & 0,1 & 0,6 & 0,9 & 0,4 & 0,6 & 1,1 & 0,5 & 0,3 \\
\hline \multicolumn{16}{|c|}{ Sector C } \\
\hline 08.Feb.11 & 0,0 & 34,0 & 0,0 & 0,0 & 0,0 & 325,0 & 18,8 & 6,7 & 0,9 & 0,1 & 0,1 & 4,0 & 2,6 & 0,0 & 1,8 \\
\hline 29.Marz.11 & 46,3 & 27,9 & 140,0 & 359,0 & 499,0 & 718,0 & 17,9 & 7,0 & 1,9 & 0,5 & 0,0 & 4,0 & 4,4 & 0,4 & 2,5 \\
\hline 11.Jul.11 & 11,9 & 32,8 & 420,0 & 117,0 & 537,0 & 234,0 & 15,8 & 6,3 & 6,2 & 0,5 & 0,0 & 9,0 & 0,6 & 0,3 & 2,0 \\
\hline 01.Ago.11 & 20,0 & 14,0 & 80,0 & 99,5 & 179,5 & 199,0 & 15,2 & 6,7 & 7,2 & 5,0 & 0,4 & 4,0 & 1,4 & 0,6 & 2,1 \\
\hline 29.Ago.11 & 0,0 & 32,0 & 60,0 & 139,0 & 199,0 & 278,0 & 16,7 & 6,2 & 6,1 & 1,9 & 0,2 & 5,0 & 0,7 & 0,2 & 2,2 \\
\hline 26.Sep.11 & 1,4 & 38,0 & 80,0 & 156,0 & 236,0 & 312,0 & 17,7 & 6,4 & 6,1 & 0,4 & 0,0 & 1,4 & 0,7 & 0,7 & 2,6 \\
\hline Promedio & 13,3 & 29,8 & 130,0 & 145,1 & 275,1 & 344,3 & 17,0 & 6,6 & 4,8 & 1,4 & 0,1 & 4,6 & 1,7 & 0,4 & 2,2 \\
\hline CV & 1,4 & 0,3 & 1,1 & 0,8 & 0,7 & 0,5 & 0,1 & 0,04 & 0,6 & 1,3 & 1,2 & 0,5 & 0,9 & 0,7 & 0,1 \\
\hline
\end{tabular}



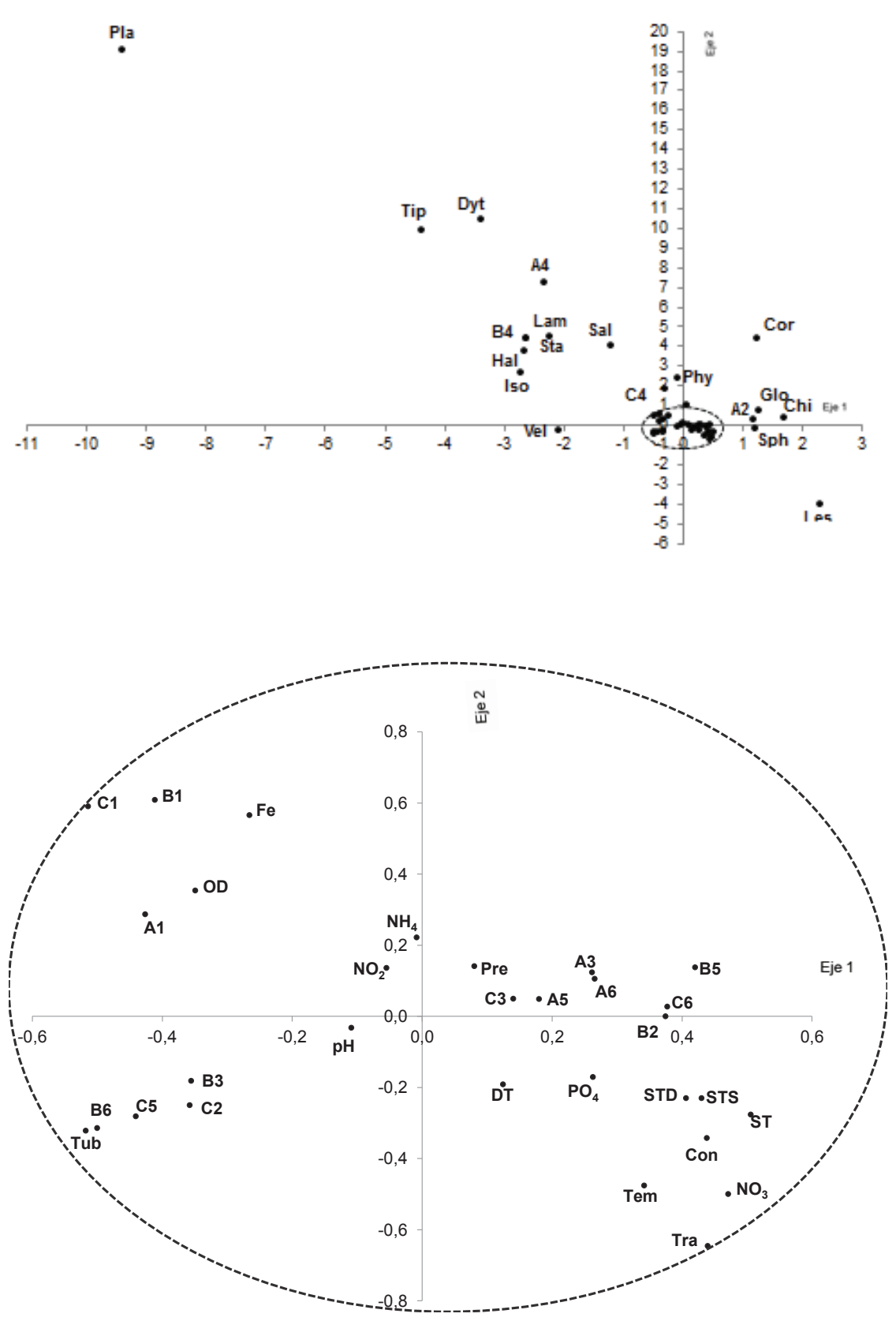

Figura 2. $A C C$ entre los tres sectores $(A B C)$ y las fechas de muestreo $(1=08$.Feb. $2=29$. Mar. $3=11$.Jul. $4=01$.Ago. $5=$ 29.Ago. $6=26$. Sep.11), las variables abióticas y las familias de macroinvertebrados bentónicos.

sólidos totales $(A=382,7 ; B=288,3 ; C=275,1)$ excedieron la concentración en aguas de la región Andina (10-200mg/L) (Roldán \& Ramírez, 2008), pero los de conductividad ( $\mathrm{A}=$ $353,8 ; \mathrm{B} ;=33,7 ; \mathrm{C}=344,3)$ revelaron aguas normales $(50$ $350 \mu \mathrm{Scm}^{-1}$ ) (APHA et al. 2005). Estos procesos también se reflejaron en el $\mathrm{O}_{2}$ disuelto, que disminuyó con las lluvias copiosas de marzo, lo que aumenta los procesos de descomposición y el consumo de $\mathrm{O}_{2}$ (Riss et al. 2002).
Según la norma, el límite para la conservación de la biota acuática es de 4mg/L (MINAMBIENTE, 2011), inferior a los promedios en los tres sectores.

Las concentraciones de $\mathrm{NH}_{4}^{+}$(0,1 y 5,0mg/l) estarían ligadas a la actividad agrícola en la cuenca alta del río Bogotá y se consideran críticas, pues $>0,5 \mathrm{mg} / \mathrm{L}$ puede ser mortal para la biota acuática (Wetzel, 2001). La normatividad en Colombia 
(MINAMBIENTE, 2011) no da un límite para el $\mathrm{NH}_{4}{ }^{+}$. Los registros más altos en el sector $\mathrm{C}$, se explican por los desagües piscícolas y de fertilizantes agrícolas, como se ha observado en otros sistemas lóticos colombianos (e.g., Ramírez et al. 2004; Sánchez, 2005; García et al. 2007; Rodríguez et al. 2007; Bustamante et al. 2008; Walteros \& Paiba, 2010). En los tres sectores, el $\mathrm{NO}_{2}{ }^{-}$promedió $0,1 \mathrm{mg} / \mathrm{L}$, por debajo de los límites legales (1,0mg/L) (MINAMBIENTE, 2011), aunque los valores más altos también se registraron en el sector $C$. Los promedios de $\mathrm{NO}_{3}{ }^{-}$fueron más altos que los de otros tramos del río Bogotá (2,7mg/L) (Roldán \& Ramírez, 2008), pero este es el compuesto nitrogenado menos tóxico (APHA et al. 2005).

Los promedios de $\mathrm{PO}_{4}{ }^{3-}$ superaron $0,15 \mathrm{mg} / \mathrm{L}$, lo que indica contaminación alta, relacionada con químicos agrícolas ingresados al agua por escorrentía e inundaciones e implica eutrofización (enriquecimiento excesivo de nutrientes), de los sistemas acuáticos (Caicedo \& Palacio, 1998).

Los promedios de pH (A: 6,3; B: 6,4; C: 6,6), se acercaron a los rangos de la mayoría de quebradas y ríos andinos $(6,5-7,5)$ y a las normas del uso del agua: 5,0-9,0 labores domésticas, 6,5-8,5 consumo humano, 4,5-9,0 actividades agropecuarias y sostenimiento de la flora y fauna asociadas (Roldán \& Ramírez, 2008). Las aguas fueron moderadamente duras (mg/L) (A: 1,9; B: 2,2; C: 1,8) , lo que refleja las características del suelo y la naturaleza geológica de las cuencas (Wetzel, 2001; Roldán \& Ramírez, 2008), mientras que el $\mathrm{Fe}^{2+}$ no sobrepasó 0,90mg/L, concentraciones muy bajas comparadas con los ríos Magdalena y Cauca (5$6 \mathrm{mg} / \mathrm{L}$ ), donde la carga de sedimentos es muy alta (Roldán \& Ramírez, 2008), como en la cuenca alta del río Bogotá (Gaviria et al. 2004).

Perfiles limnológicos similares al obtenido en el área de estudio, reportaron Sierra et al. (2002), para el mismo tramo del río Bogotá y Gaviria et al. (2004), para el sector de Checua, también ubicado en la cuenca alta del río Bogotá.

Correlación entre variables: El ACC detectó diferencias entre sectores, pues solamente se agruparon los tres el 08.Feb.11 y el 01.Ago.11 y, parcialmente, el 11.Jul.11 (A y C) y el 29.Ago.11 (A y B), con mayores asociaciones positivas de las dos últimas fechas con las precipitaciones y negativas con la transparencia, sólidos en general, conductividad,

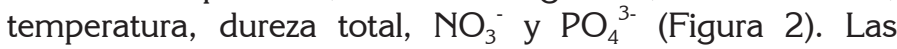
diferencias según la época de muestreo, se atribuyeron

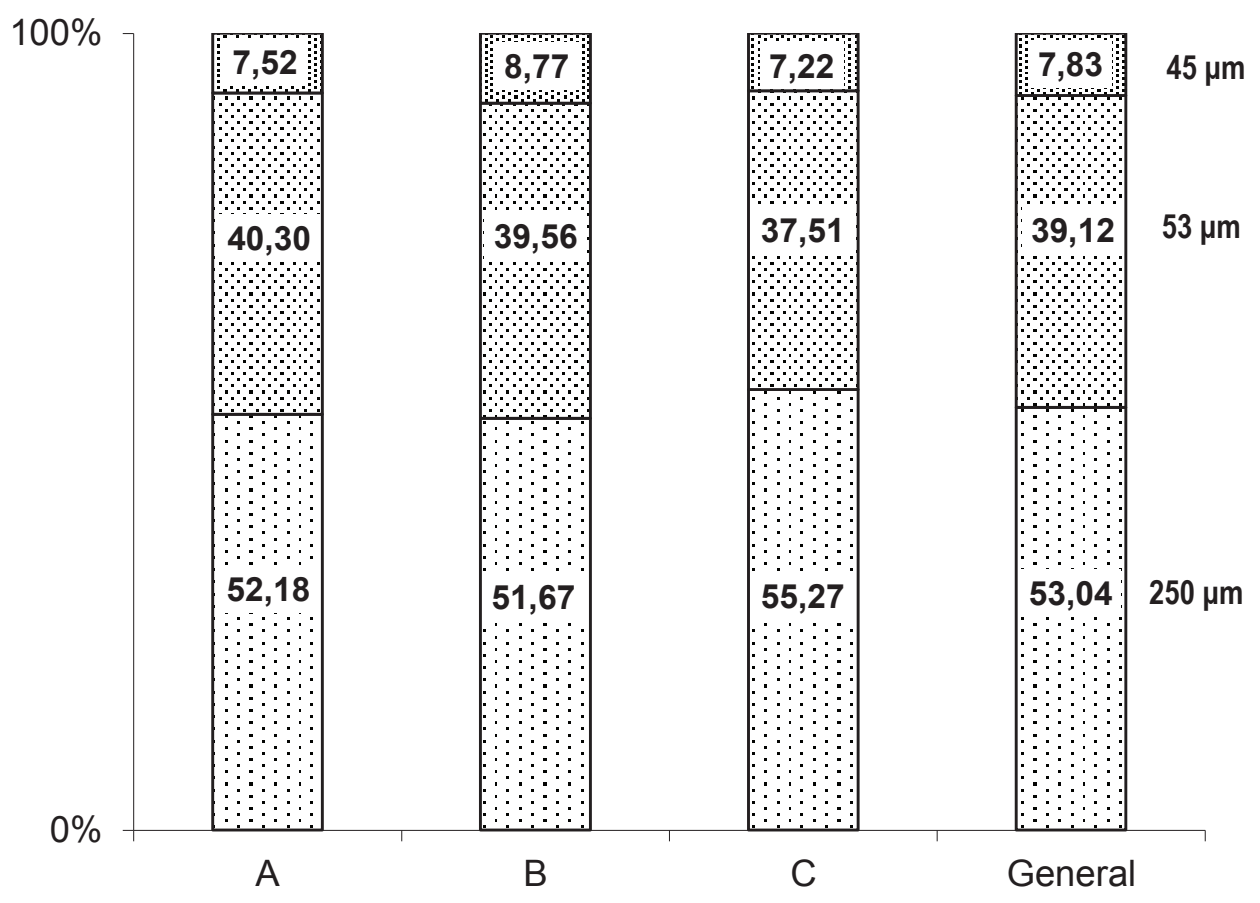

Figura 3. Composición porcentual del sustrato en los tres sectores y en general en el tramo del río Bogotá evaluado, según la escala de Wentworth (Wetzel, 2001). 
a la influencia de las lluvias, que incluye procesos de mineralización (Riss et al. 2002) y a las entradas temporales de material alóctono, i.e., vertimientos de aguas negras y con remanentes de actividades agropecuarias, comunes en la cuenca alta del río Bogotá (Sierra et al. 2002; CARCundinamarca, 2006).

El ACC también reveló el 01.Ago.11 mayor asociación entre los tres sectores y ocho de las 17 familias identificadas, correspondiente con los valores más altos del BMWP/Col. (41) y ASPT (5), i.e., el mayor grado de contaminación dentro de las fechas consideradas.

Análisis del sustrato: Se encontró homogeneidad del sustrato entre los tres sectores, donde primaron la arena fina $(53,0 \%)$ y el limo (39,1\%) (Figura 3), lo que obedece a su remoción constante de rocas areniscas y arcillosas en la cuenca alta del río Bogotá, lo que ocasiona una carga sedimentaria alta por la explotación minera y los arados constantes en los alrededores del río; la minería también genera impactos ecológicos, como el desplazamiento masivo y lento del suelo, al absorber gran cantidad de agua (CAR-Cundinamarca, 2006). Hay similitud con los ríos Cauca (Ramírez et al. 2004), Atrato y León (Álvarez \& Bernal, 2007), donde predominan las arenas medias, finas y gruesas.

Conflictos de intereses. El manuscrito fue preparado y revisado con la participación de todos los autores, quienes declaramos que no existe ningún conflicto de intereses que ponga en riesgo la validez de los resultados presentados. Financiación. Este estudio fue financiado por la Vicerrectoría de Investigaciones y el Laboratorio de Hidrobiología de la Universidad Militar Nueva Granada.

\section{BIBLIOGRAFÍA}

1. ÁlVAREZ, A.; BERNAL, G. 2007. Estimación del campo de transporte neto de sedimentos en el fondo de bahía Colombia con base en análisis de tendencia del tamaño de grano. Escuela de Geociencias y Medio Ambiente, Universidad Nacional de Colombia (Medellín). Avances Rec. Hidr. 16:41-50.

2. APHA, AWWA y WPCF. 2005. Standard methods for the examination of water and wastewater. American Public Health Association (APHA), American Water Works Association (AWWA) and Water Environment Federation (WEF) (Washington). 1325p.

3. ARANGO, M.C.; ÁLVAREZ, L.F.; ARANGO, G.A.; TORRES, O.E.; MONSALVE, A. DE J. 2008. Las quebradas la Cristalina y la Risaralda, San Luis, Antioquia. Rev. EIA. 9:121-141.
4. ARMITAGE, P.D.; MOSS, D.; WRIGHT, J.F.; FURSE, M.T. 1983. The performance of a new biological water quality score based on macroinvertebrates over a wide range of unpolluted running-water sites. Wat. Res. 17:333-347.

5. BUSTAMANTE, C.A.; MONSALVE, E.; GarcíA, P.L. 2008. Análisis de la calidad del agua en la cuenca media del río Quindío con base en índices físicos, químicos y biológicos. Rev. Invest. U. Quindío. 18:22-31

6. CAICEDO,O.;PALACIO,J.1998. Losmacroinvertebrados bentónicos y la contaminación orgánica en la quebrada la Mosca (Guarne Antioquia, Colombia). Act. Biol. 20(69):61-73.

7. CAR-Cundinamarca. 2006. Plan de ordenación y manejo de la cuenca hidrográfica del río Bogotá. Resumen ejecutivo. Corporación Autónoma Regional de Cundinamarca (CAR) (Bogotá, D. C.). 104p.

8. CASTELLANOS, P.M.; SERRATO, C. 2008. Diversidad de macroinvertebrados acuáticos en un nacimiento de río en el páramo de Santurbán, Norte de Santander. Rev. Acad. Colomb. Cienc. 32(122):79-86.

9. GARCÍA, C.; ROMÁN, C.; VANEGAS, J.; ARCILA, D. 2007. Análisis fisicoquímico y biológico comparado en dos quebradas de alta montaña neotropical. Rev. Invest. U. Quindío. 17:57-80.

10. GARCÍA, J.; CARMONA, J.C.; MONTOYA, Y. 2012. Caracterización de la calidad del agua de la parte alta de la microcuenca de la quebrada La Cimarronas, El Carmen de Viboral (Antioquia), utilizando macroinvertebrados acuáticos. Inv. Biodiv. y Des. 31(1):5-18.

11. GAVIRIA, S.; HERNÁNDEZ, O.; VARGAS, O. 2004. Relación entre procesos de erosión y geoquímica de sedimentos y suelos de Checua, cuenca alta del río Bogotá. Rev. Acad. Col. Cienc. 28(109):497-508.

12. GOITIA, E.; BUSTAMANTE, M. 2009. Patrones espaciales de los macroinvertebrados bentónicos en la región de Yungas de Cochabamba (Bolivia). Rev. Bol. Ecol. y Cos. Amb. 26:25-43.

13. IDEAM. 2011. Boletín informativo sobre el Monitoreo del Fenómeno de "LA NIÑA", Instituto de Hidrología, Meteorología y Estudios Ambientales (IDEAM). 32:16. 
14. JACOBSEN, D. 2003. Altitudinal changes in diversity of macroinvertebrates from small streams in the Ecuadorian Andes. Arch. Hydrobiol. 158(2):145167.

15. JONES, N.E. 2011. Benthic sampling in natural and regulated rivers. Sampling methodologies for Ontario's flowing waters. Ontario Ministry of Natural Resources, Aquatic Research and Development Section, River and Stream Ecology Lab, Aquatic Research Series 2011-05, Ontario. 16p.

16. LIÉVANO, A.; OSPINA, R. 2007. Guía ilustrada de los macroinvertebrados acuáticos del río Bahamón. Bogotá D.C. Universidad El Bosque e Instituto Alexander Von Humbolt. Bogotá, D. C. 130p.

17. LOZANO, L.O. 2005. La bioindicación de la calidad del agua: Importancia de los macroinvertebrados en la cuenca alta del Río Juan Amarillo, Cerros Orientales de Bogotá. Umb. Cient. 7:5-11.

18. MARÍN, N.C.; CORREDOR, N.V.; BUSTAMANTE, C.A. 2011. Aspectos ecológicos de comunidades bénticas de un sistema fluvial andino, Armenia-Quindío, Colombia. Rev. Invest. Univ. Quindío. (22):58-69.

19. MERRIT, R.; CUMMINS, K. 1984. An introduction to the aquatic insects of North America. Kendall/Hall Publishing Company (Dubuque, lowa). 722p.

20. MILÁN, W.Y.; CAICEDO, V.O.; AGUIRRE N.J. 2011. Quebrada La Popala: un análisis de calidad del agua desde algunas variables fisicoquímicas. Gest. y Amb. 14(1):85-94.

21. MINAMBIENTE. 2011. Guía metodológica de trámites para el control de los vertimientos en los cuerpos de agua superficiales, al suelo asociado a un acuífero y al medio marino, en función de los trámites y procedimientos requeridos para la obtención de los permisos de vertimientos. Ministerio de Ambiente y Desarrollo Sostenible (MINAMBIENTE). Dirección Gestión Integral del Recurso Hídrico. Bogotá, D. C. $54 p$.

22. MUGNAI, R.; NESSIMIAN, J.; FERNANDEZ, D. 2010. Manual de identificacao de Macroinvertebrados aquáticos do Estado do Rio de Janeiro. Tecnical Books Ed. (Rio de Janeiro). 176p.

23. MONTOYA, Y.M. 2008. Caracterización de la biodiversidad acuática y de la calidad de las aguas de la quebrada Los Andes, El Carmen de Viboral, Antioquia. Inv., Biodiv. Des. 27(1):85-91.

24. MONTOYA, Y.; ACOSTA, Y.; ZULUAGA, E. 2011. Evolución de la calidad del agua en el río Negro y sus principales tributarios empleando como indicadores los índices ICA, el BMWP/COL y el ASPT. Caldasia. 33(1):193-210.

25. OYAGUE, E. 2009. Discusión sobre el número de unidades muestrales y tamaño de unidad muestral para la estimación de valores de densidad de macroinvertebrados bentónicos en ambientes lóticos. Ecol. Apl. 8(1-2):61-70.

26. RAMÍREZ, C.; GARCÍA, J.; RAMÍREZ, O.; BOCANEGRA, R. 2004. Caracterización de los materiales del lecho del río Cauca. Facultad de Ingeniería, Universidad del Valle. Ing. Rec. Nat. Amb. 1(1):10-17.

27. RISS, W.; OSPINA, R.; GUTIÉRREZ, J. 2002. Establecimiento de valores de bioindicación para los macroinvertebrados acuáticos de la Sabana de Bogotá. Caldasia. 24(1):135-156.

28. RODRÍGUEZ, J.; OSPINA, R.; GUTIÉRREZ, J.; OVALLE, H. 2007. Densidad y biomasa de macroinvertebrados acuáticos derivantes en una quebrada tropical de montaña (Bogotá, Colombia). Caldasia. 29(2):397412.

29. ROLDÁN, G. 1996. Guía para el estudio de los macroinvertebrados acuáticos del departamento de Antioquia. FEN Colombia. COLCIENCIAS-UDEA (Medellín). 217p.

30. ROLDÁN, G. 2003. Bioindicación de la calidad del agua en Colombia. propuesta para el uso del método BMWP/Col. Ed. Universidad de Antioquia (Medellín). 170p.

31. ROLDÁN, G.; RAMÍREZ, J. 2008. Fundamentos de limnología neotroropical. Ed. Universidad de Antioquia (Medellín). 440p.

32. SÁNCHEZ, M. 2005. El índice biológico BMWP (Biological Monitoring Working Party Score), modificado y adaptado al cauce principal del río Pamplonita Norte de Santander. Bistua. (2):54-67.

33. SIERRA, J.; JAIME, A.; MORA, A. 2002. Monitoreo de parámetros físico-químicos, en la cuenca alta del río Bogotá. Rev. Cienc. Ing. Neogran. 12:23-30. 
34. WALLEY, W. J.; HAWKES H. A. 1997. A Computer based development of the biological monitoring working party score system incorporating abundance rating, site type and indicator value. Wat. Res. 31(2):201210.

35. WALTEROS, J.M.; PAIBA, J.E. 2010. Estudio preliminar de la comunidad de macroinvertebrados acuáticos en la Reserva Forestal Torre Cuatro. Bol. Cient. Mus. Hist. Nat. 14(1):137-149.

36. WETZEL, R.G. 2001. Limnology. Lake and river ecosystems. Academic Press (San Diego). 1006p.

Recibido: Septiembre 14 de 2012

Aceptado: Febrero 21 de 2013 